\title{
Optimal Excitation of Baroclinic Waves in the Eady Model
}

\author{
By Hitoshi Mukougawa ${ }^{1}$ and Toru Ikeda ${ }^{2}$
}

Meteorological College, Kashiwa 277, JAPAN

(Manuscript received 4 October 1993, in revised form 28 February 1994)

\begin{abstract}
In the framework of the linear theory by using the Eady model, properties of optimal baroclinic perturbations which attain the maximum growth over a prescribed time interval $(\tau)$ are examined in connection with the skill forecast problem in numerical weather prediction (NWP). The development of the optimal perturbation depends not only on the linear stability of the basic flow, but also on the time interval $\tau$ to assess its growth.

In the unstable region, the optimal perturbation grows almost exponentially in time as the unstable normal mode. Its structure gradually coincides with the unstable normal mode as $\tau$ becomes large. The corresponding optimal initial disturbance becomes the adjoint of the unstable mode. For smaller $\tau(\tau \leq 3$ days), however, the phase line of the optimal perturbation has a larger inclination than the unstable normal mode, which plays a negligible role in this early development.

In the neutral region, the maximum amplitude of the optimal perturbation grows algebraically in time as $C \tau^{2}+1$ ( $C$ is a constant). The optimal initial disturbance has a plane wave structure leaning against the shear. The phase line becomes more horizontal and the vertical scale decreases inversely with $\tau$. On the other hand, the excited optimal perturbation has an almost barotropic structure, and is composed mainly of the two neutral non-singular normal modes with different phase speeds. The growth of the optimal perturbation is quantitatively well understood by the Orr mechanism for larger $\tau$ ( $\tau \geq 2$ days). For shorter $\tau$, however, the interference between the two neutral non-singular normal modes has a major contribution to the development. The structure of the excited optimal perturbation is also explained by considering the projectability for each mode.

This study suggests that a high resolution model which is comparable to the operational full NWP model is necessary to predict accurately the forecast skill of the NWP model.
\end{abstract}

\section{Introduction}

Since the pioneering work of Charney (1947) and Eady (1949), many studies have been devoted to reveal the dynamics of migrating synoptic scale disturbances in mid-latitudes by the baroclinic instability theory. In this traditional stability analysis, the linearized perturbation is assumed to have a fixed normal mode structure and to grow exponentially in time. We concentrate on finding the most unstable mode with the largest growth rate. However, the structure of the initial disturbance can be disregarded since the most unstable mode will inevitably dominate over the structure of the disturbance field in an infinite time interval. The baroclinic instability theory has been confirmed in its success by predicting the horizontal scale, phase speed, and struc-

1 Present affiliation: Graduate School of Environmental Earth Science, Hokkaido University, Sapporo 060, JAPAN

2 Present affiliation: Fukui Meteorological Observatory, Fukui 910, JAPAN

(C)1994, Meteorological Society of Japan ture of the synoptic scale disturbance with the most unstable mode in the basic baroclinic shear flow.

On the other hand, Farrell (1982) pointed out that perturbations not of a normal mode form undergo transient development on time scales appropriate to synoptic scales and that the growth rate of these favorable perturbations can greatly exceed that of the most unstable mode in the context of the linear theory. The so-called continuous spectra of neutral modes (Pedlosky, 1987), which form a complete set together with discrete normal modes in order to represent an arbitrary initial condition, have an important role in the transient development of disturbances. Moreover, Farrell $(1988,1989)$ formulated the optimization analysis in barotropic and baroclinic shear flows in order to determine the structure of the optimal linear perturbation which attains the largest wave amplitude at the end of a prescribed finite time interval. The obtained optimal perturbation does not have a normal mode structure and its amplitude does not grow exponentially in time. In this analysis, the corresponding optimal initial 
disturbance which excites the optimal perturbation after a prescribed time interval must be also specified.

The role of the optimal perturbation was originally discussed in connection with the explosive cyclogenesis (Farrell, 1989). Recently, many researchers have been aware of its importance in deciding the local or finite-time predictability of the atmospheric motions, although the research on this issue can be traced back to the original work of Lorenz (1965). Farrell (1990) discussed the predictability of baroclinic and barotropic shear flows with idealized configurations, while Borges and Hartmann (1992) obtained optimal perturbations for observed non-zonal flows by using a barotropic model. It is also possible to extend the optimization analysis for the steady basic flow to that for the time-dependent basic flow by carrying out time integrations of the tangent linear equations about the time-varying basic state (Lacarra and Talagrand, 1988; Mukougawa et al., 1991; Kimoto et al., 1992). Yoden and Nomura (1993) gave a well arranged instructive review on this subject by discussing the relation with the local or finite-time Lyapunov stability analysis. More recently, Molteni and Palmer (1993) and Mureau et al. (1993) performed optimization analysis to generate effective perturbations for ensemble forecasts by using the numerical weather prediction (NWP) model at ECMWF (see also, Palmer, 1993; Toth and Kalnay, 1993).

Although the studies cited above have succeeded in designating the important role of the optimal perturbation to determine the local predictability, the physical interpretation for the structure of the optimal perturbation and the mechanism for its growth have not been fully elucidated as yet. Moreover, whether the fine structure of the optimal initial disturbance, which is commonly seen in these studies [e.g., see Fig. 7 in Yoden and Nomura (1993)], is affected by the spatial resolution of the model has not been clarified.

In this paper, we study the detailed properties of the optimal baroclinic perturbation in the context of the linear theory. We pay our attention to the dependence on the stability of the basic flow and the prescribed time interval to assess its growth, and try to clarify the growing mechanism of the optimal perturbation. For this aim, we use the Eady model (Eady, 1949), which has elegantly exhibited the baroclinic instability in its purest form. Furthermore, we address the sensitivity of the structure of the optimal perturbation to the model resolution. From this result, we can assess the required spatial resolution of the NWP model to represent faithfully the growth of the optimal perturbation.

We describe the model in Section 2. Section 3 gives the optimization analysis in comparison with the traditional stability analysis. The results are given in Section 4. Section 5 is for discussions on the physical interpretation of the optimal perturbation. In particular, the growth of the optimal perturbation in the neutral region is quantitatively discussed in comparison with the growth inferred from the Orr mechanism (Orr, 1907; Boyd, 1983; Lindzen, 1990). The vertical structure of the disturbance is also examined by taking account of the projectability (Borges and Hartmann, 1992) for each mode. Conclusions are presented in Section 6.

\section{Model}

We consider the temporal evolution of linear perturbations in the Eady model (Eady, 1949). In this model, the basic flow $U_{0}$ is assumed to have a constant vertical shear $\lambda$. The fluid is confined in a channel with two rigid horizontal boundaries in the vertical direction. Assuming a constant static stability, a constant density of the basic state (the Boussinesq approximation), and neglecting the beta effect, the non-dimensional linearized perturbation quasigeostrophic vorticity equation becomes (Pedlosky, 1987)

$$
\left(\frac{\partial}{\partial t}+U_{0} \frac{\partial}{\partial x}\right) \nabla^{2} \psi=\frac{\partial w_{1}}{\partial z}
$$

where the basic flow $U_{0}=\lambda z, \psi(x, y, z, t)$ is the geostrophic streamfunction, and $w_{1}$ the ageostrophic vertical velocity. The horizontal Laplacian operator is defined as $\nabla^{2}=\partial^{2} / \partial x^{2}+\partial^{2} / \partial y^{2}$. The linearized thermodynamic equation is

$$
\left(\frac{\partial}{\partial t}+U_{0} \frac{\partial}{\partial x}\right) \frac{\partial \psi}{\partial z}-\frac{\partial U_{0}}{\partial z} \frac{\partial \psi}{\partial x}+S w_{1}=0
$$

where $S$ is the static stability parameter. Vertical boundary conditions are given by

$$
w_{1}=0, \text { at } z=0,1 .
$$

Since two rigid walls of the channel are also placed at $y= \pm 1$, the lateral boundary conditions are

$$
\frac{\partial \psi}{\partial x}=0, \text { at } y= \pm 1
$$

All variables and constants are non-dimensionalized: $x$ and $y$ by $L ; z$ by $D ; t$ by $L / U ; \psi$ by $L U$; $w_{1}$ by $D U^{2} /\left(f_{0} L^{2}\right)$. The static stability parameter $S$ is defined as $S=N_{s}^{2} D^{2} /\left(f_{0}^{2} L^{2}\right)$ where $N_{s}$ is the Brunt-Väisälä frequency. We choose these parameter values which are appropriate to the mid-latitude synoptic scale disturbance in the troposphere (Pedlosky, 1987): the horizontal scale $L=2 \times 10^{6} \mathrm{~m}$, the vertical scale $D=10^{4} \mathrm{~m}$, the horizontal velocity $U=10 \mathrm{~ms}^{-1}$, the Coriolis parameter $f_{0}=10^{-4}$ $\mathrm{s}^{-1}$, and $N_{s}=10^{-2} \mathrm{~s}^{-1}$. With these values, the static stability parameter becomes $S=0.25$. The meridional width of this channel model is $4000 \mathrm{~km}$ and a unit of non-dimensionalized time corresponds to $55.6 \mathrm{~h}$ (2.3 days). 
Eliminating $w_{1}$ in (1) with (2), we obtain the perturbation potential vorticity equation:

$$
\left(\frac{\partial}{\partial t}+U_{0} \frac{\partial}{\partial x}\right)\left(\nabla^{2} \psi+\frac{1}{S} \frac{\partial^{2} \psi}{\partial z^{2}}\right)=0
$$

Thus, the perturbation potential vorticity $q$ defined by

$$
q \equiv \nabla^{2} \psi+\frac{1}{S} \frac{\partial^{2} \psi}{\partial z^{2}}
$$

is conserved following the particle motion.

In order to solve the optimization problem, we use the following $N$-layer representation. There are $N$ interior levels at $z_{i}=(2 i-1) / 2 N(i=1,2, \cdots, N)$. The vorticity equation (1) is applied at the interior levels $z_{i}(i=1,2, \cdots, N)$, and the thermodynamic equation (2) at the intermediate levels $z_{i+1 / 2}=i / N$ $(i=1,2, \cdots, N-1)$. The boundary conditions (3) are incorporated at the levels $z_{1 / 2}=0$ and $z_{N+1 / 2}=$ 1.

It is also assumed that the streamfunction $\psi_{i}(x, y, t)$ at the level $z_{i}$ has a sinusoidal wave form:

$$
\psi_{i}(x, y, t)=\operatorname{Re}\left[\hat{\psi}_{i}(t) \mathrm{e}^{i k x}\right] \cos l_{n} y
$$

where $\operatorname{Re}[\bullet]$ denotes the real part in the bracket, $k$ the zonal wavenumber, and the lateral wavenumber $l_{n}$ must be

$$
l_{n}=\left(n+\frac{1}{2}\right) \pi, \quad n=0,1,2, \cdots,
$$

in order to satisfy the lateral boundary conditions (4). In this study, the gravest meridional mode with $n=0$, which gives the most unstable mode structure (Pedlosky, 1987), is assumed. Eliminating the perturbation vertical velocity $w_{1}$ from the discretized equations, we finally obtain a set of $N$ ordinary differential equations for $\hat{\psi}_{i}(t)$ the complex amplitude of the streamfunction at $z_{i}$, which can be symbolically written as

$$
\mathrm{B} \frac{d}{d t} \hat{\boldsymbol{\psi}}=\mathrm{D} \hat{\boldsymbol{\psi}}
$$

where $\hat{\boldsymbol{\psi}}=\left(\hat{\psi}_{1}, \hat{\psi}_{2}, \cdots, \hat{\psi}_{N}\right)^{\top}, \mathrm{B}$ and D are $N \times N$ matrices.

\section{Formulation}

\section{a. Stability problem}

If we assume a normal mode form as the perturbation, i.e.,

$$
\hat{\psi}_{i}(t)=\phi_{i} \mathrm{e}^{-i k c t}
$$

with the complex phase speed $c$, a set of $N$ homogeneous linear algebraic equations for $\phi=\left(\phi_{1}, \phi_{2}\right.$, $\left.\cdots, \phi_{N}\right)^{\top}$ is obtained from $(9)$,

$$
\lrcorner \phi=c \phi
$$

where $\mathrm{J}=i \mathrm{~B}^{-1} \mathrm{D} / k$ is the $N \times N$ Jacobian matrix. Thus, the stability of the basic flow $U_{0}$ is determined by solving the eigenvalue problem of the matrix $\mathrm{J}$. If there is any eigenvalue $c$ with a positive imaginary part $(\operatorname{Im}[c]>0)$, the basic flow is unstable for an infinitesimal perturbation. The instability theory also predicts that the most unstable mode with the largest growth rate $\operatorname{Im}[k c]$ will emerge first from the background of small disturbances and dominate over the disturbance field in an infinite time interval.

\section{b. Temporal evolution of an arbitrary initial dis- turbance}

Before formulating the optimization problem, it is necessary to specify the temporal evolution of an arbitrary initial disturbance. Since each normal mode $\phi^{i}$ with a complex phase speed $c^{i}(i=1, \cdots, N)$ obtained from the eigenvalue problem of (11) is linearly independent when the phase speed is different for each mode, i.e., $c^{i} \neq c^{j}$ for $i \neq j$, the evolution of the complex amplitude $\hat{\psi}(t)$ is written by the summation over all modes:

$$
\hat{\boldsymbol{\psi}}(t)=\sum_{i=1}^{N} \alpha^{i} \phi^{i} \exp \left(-i k c^{i} t\right),
$$

where $\alpha^{i}$ is the projection coefficient for the mode $i$. If two eigenvalues coalesce, the solution to the initial value problem cannot in general be written as a sum over separable modes of the form (12) since the corresponding two eigenvectors are linearly dependent (Held, 1985). In such a case, the initial value problem must be solved by using a combined Fourier-Laplace integral, as was shown by Pedlosky (1964) in the Eady model. Unfortunately, the inversion of these integrals is a formidable task and results are obtained for the asymptotic limit of long time only (see also Farrell, 1982).

In obtaining $\alpha^{i}$, we use the following biorthogonality of $\phi^{i}$ :

$$
\left\langle\phi^{i} \cdot \tilde{\phi}^{j}\right\rangle \equiv \sum_{k=1}^{N} \phi_{k}^{i}\left(\tilde{\phi}_{k}^{j}\right)^{*}=0, \text { for } i \neq j,
$$

where the asterisk denotes the complex conjugate. The adjoint mode $\tilde{\phi}^{j}$ satisfies the adjoint eigenvalue problem:

$$
\mathrm{J}^{\mathrm{T}} \tilde{\phi}^{j}=\left(c^{j}\right)^{*} \tilde{\phi}^{j}
$$

where $\mathrm{J}^{\top}$ is the adjoint matrix of $\mathrm{J}$. Thus, the projection coefficient $\alpha^{i}$ is given by

$$
\alpha^{i}=\frac{\left\langle\hat{\psi}(0) \cdot \tilde{\phi}^{i}\right\rangle}{\left\langle\phi^{i} \cdot \tilde{\phi}^{i}\right\rangle} .
$$

Furthermore, the perturbation streamfunction $\psi(x, y, t)=\left(\psi_{1}, \psi_{2}, \cdots, \psi_{N}\right)^{\top}$ in (7) is rewritten 
as

$$
\begin{aligned}
\psi(x, y, t) & =\operatorname{Re}\left[\sum_{i=1}^{N} \alpha^{i} \phi^{i} \exp i k\left(x-c^{i} t\right)\right] \cos l_{n} y \\
& =\left(\boldsymbol{\psi}_{c}(t) \cos k x+\boldsymbol{\psi}_{s}(t) \sin k x\right) \cos l_{n} y
\end{aligned}
$$

Thus, the evolution of the perturbation streamfunction is determined by the $2 N$ dimensional real vector $\boldsymbol{y}(t)$,

$$
\boldsymbol{y}(t)=\left(\begin{array}{c}
\boldsymbol{\psi}_{c}(t) \\
\boldsymbol{\psi}_{s}(t)
\end{array}\right)
$$

From (16), the vectors $\psi_{c}$ and $\psi_{s}$ at $t=0$ and $t=\tau$ are given by

$$
\boldsymbol{\psi}_{c}(0)=\operatorname{Re}[\boldsymbol{\Phi} \boldsymbol{\alpha}], \quad \boldsymbol{\psi}_{s}(0)=-\operatorname{Im}[\boldsymbol{\Phi} \boldsymbol{\alpha}],
$$

and

$$
\boldsymbol{\psi}_{c}(\tau)=\operatorname{Re}[\boldsymbol{\Phi} \boldsymbol{\Lambda} \boldsymbol{\alpha}], \quad \boldsymbol{\psi}_{s}(\tau)=-\operatorname{Im}[\boldsymbol{\Phi} \boldsymbol{\Lambda} \boldsymbol{\alpha}],
$$

where $\boldsymbol{\Phi}$ is an $N \times N$ matrix with the eigenvectors $\phi^{i}$ as columns, $\boldsymbol{\Lambda}$ is an $N \times N$ diagonal matrix with $\exp \left(-i k c^{i} \tau\right)$ as elements, and $\boldsymbol{\alpha}=\left(\alpha^{1}, \alpha^{2}, \cdots\right.$, $\left.\alpha^{N}\right)^{\top}$. Eliminating the vector $\boldsymbol{\alpha}$ from (18) and (19), the perturbation $\boldsymbol{y}(\tau)$ at time $\tau$ is related with the specified initial disturbance $\boldsymbol{y}(0)$ by

$$
\boldsymbol{y}(\tau)=\mathrm{M}(\tau) \boldsymbol{y}(0) .
$$

Here, $2 N \times 2 N$ matrix $\mathrm{M}(\tau)$, which is defined as

$$
\mathrm{M}(\tau)=\left(\begin{array}{cc}
\operatorname{Re}\left[\boldsymbol{\Phi} \boldsymbol{\Lambda} \boldsymbol{\Phi}^{-1}\right] & \operatorname{Im}\left[\boldsymbol{\Phi} \boldsymbol{\Lambda} \boldsymbol{\Phi}^{-1}\right] \\
-\operatorname{Im}\left[\boldsymbol{\Phi} \boldsymbol{\Lambda} \boldsymbol{\Phi}^{-1}\right] & \operatorname{Re}\left[\boldsymbol{\Phi} \boldsymbol{\Lambda} \boldsymbol{\Phi}^{-1}\right]
\end{array}\right),
$$

determines the evolution during the time interval $\tau$. Note that $\mathrm{M}(\tau)$ is obtained by eigenvalues and eigenvectors of the matrix $\mathrm{J}$.

\section{c. Optimization problem}

In this study, we examine the optimal perturbation which maximizes $\|\boldsymbol{y}(\tau)\|$ the $L_{2}$ norm of the vector $\boldsymbol{y}(\tau)$ corresponding to the amplitude of the perturbation streamfunction after a prescribed time interval $\tau$. Hereafter, we will refer to $\|\boldsymbol{y}(\tau)\|$ as the "amplitude" of the perturbation. The procedure to obtain the optimal perturbation is well understood by considering the evolution of isotropic random initial disturbances at $t=0$, which are represented by a $2 N$-dimensional sphere with unit radius (Lorenz, 1965; Yoden and Nomura, 1993):

$$
\boldsymbol{y}^{\top}(0) \boldsymbol{y}(0)=1 \text {. }
$$

According to (20), this sphere will be deformed into an ellipsoid at $t=\tau$ :

$$
\boldsymbol{y}^{\top}(\tau)\left[\mathrm{M}(\tau) \mathrm{M}^{\top}(\tau)\right]^{-1} \boldsymbol{y}(\tau)=1
$$

Since the eigenvalue $\Gamma_{i}$ and the corresponding normalized eigenvector $\boldsymbol{\zeta}_{i}$ of the symmetric matrix $\mathrm{M}(\tau) \mathrm{M}^{\top}(\tau)$ give the square length and the direction of the $i$-th largest semiaxis of this ellipsoid, the spatial structure of the optimal perturbation is given by $\zeta_{1}$ with the largest eigenvalue $\Gamma_{1}$, while its amplitude is $\Gamma_{1}^{1 / 2}$.

On the other hand, the initial disturbance which excites the optimal perturbation after time $\tau$ is given by the eigenvector $\boldsymbol{f}_{1}$ with the largest eigenvalue $\Gamma_{1}$ of the matrix $\mathrm{M}^{\top}(\tau) \mathrm{M}(\tau)$ (Yoden and Nomura, 1993). The eigenvectors $\boldsymbol{f}_{i}$ form an orthonormal set as $\boldsymbol{\zeta}_{i}$. Note also that both of the optimal perturbation $\boldsymbol{\zeta}_{1}$ and the corresponding optimal initial disturbance $\boldsymbol{f}_{1}$ depend on the time interval $\tau$ to assess the growth as well as the stability of the basic state.

We define the "growth rate" of the optimal perturbation for the time interval $\tau$ by $\ln \Gamma_{1} /(2 \tau)$, which corresponds to the average amplification rate over $\tau$. In the context of the predictability, this value is referred to as the finite-time largest Lyapunov exponent, and the optimal initial disturbance $\boldsymbol{f}_{1}$ is the corresponding finite-time Lyapunov vector (Yoden and Nomura, 1993).

\section{Results}

\section{a. Sensitivity of the growth rate to the vertical resolution}

The obtained growth rate of the optimal perturbation inevitably depends on the vertical resolution, i.e., the total layer number $N$ of the model. Thus, we examine the sensitivity of the growth rate of the optimal perturbation to the total layer number $N$ in order to assure our results. Figure 1 shows how the growth rate of the optimal perturbation depends on $N(2 \leq N \leq 100)$ for some parameter values (see also Figs. 2 and 3 ). The growth rate monotonically increases with the increase of $N$, but the convergence is rather weak compared with the rapid convergence of the growth rate of the most unstable mode; the 6-layer model is sufficient to fully resolve the most unstable mode in the Eady problem (Bell and White, 1988). It is also found that the convergence of the growth rate of the optimal perturbation becomes worse with the increase of the zonal wavenumber $k$ (compare $\times$ with $\bigcirc$ ), the increase of the vertical shear $\lambda$ of the basic flow (compare $\bigcirc$ with $\square$ ), and the increase of the prescribed time interval $\tau$ (compare $\bigcirc$ with $\triangle$ ). This is because the vertical structure of the optimal initial disturbance intimately relates to these parameters, which will be discussed later. In this study, we will use the 50 -layer model which performs an allowable convergence of the growth rate for synoptically relevant time scales: the growth rate in the 50-layer model attains over $98 \%$ of that in the 100-layer model. 


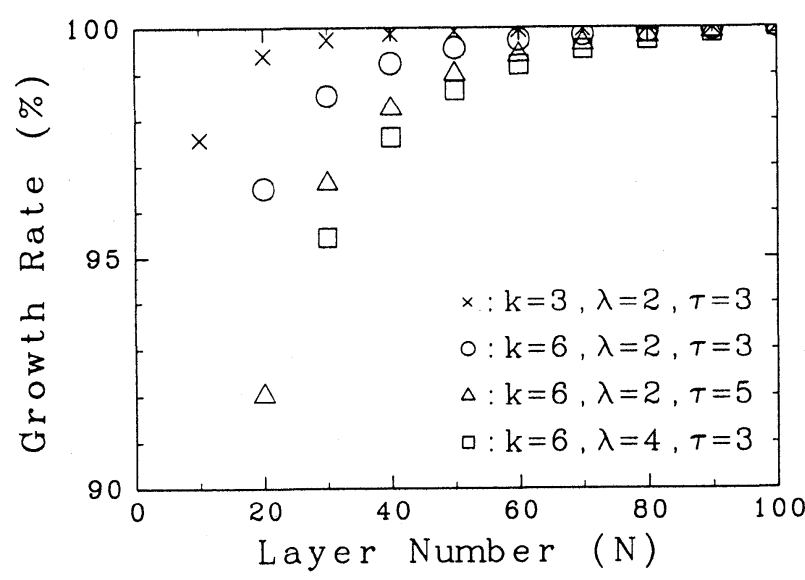

Fig. 1. Sensitivity of the growth rate of the optimal perturbation to the vertical resolution of the model. The abscissa indicates the total layer number $N$ of the model and the ordinate the ratio of the growth rate in the $N$-layer model to that in the 100-layer model: $\times$ is for $k=3$, $\lambda=2$, and $\tau=3 ; \bigcirc$ for $k=6, \lambda=2$, and $\tau=3 ; \triangle$ for $k=6, \lambda=2$, and $\tau=5 ; \square$ for $k=6, \lambda=4$, and $\tau=3$.

\section{b. Growth rate of the most unstable mode}

Figure 2 shows the well known stability diagram for the Eady model with adopted parameter values. The growth rate of the most unstable mode is contoured by lines in $k-\lambda$ section. The basic flow is unstable for perturbations with small zonal wavenumbers $(k \leq 4.53)$, and the maximum growth rate attains at $k=3.05$. The growth rate is proportional to the vertical shear $\lambda$ of the basic flow. However, the critical line for the instability and the wavenumber of the most unstable mode do not depend on the vertical shear $\lambda$.

Note that the eigenvalues in the $N$-layer Eady model are classified into two non-singular normal modes and $N-2$ singular normal modes. The nonsingular normal mode corresponds to either the unstable or stable mode in the unstable region, while it corresponds to either of the two neutral modes with different phase speeds in the neutral region. The singular mode, which has a delta-function singularity at its steering level in the perturbation potential vorticity $q$ of (6), corresponds to the neutral continuous spectrum of eigenvalues (Farrell, 1982; Pedlosky, 1987).

The eigenvalue of the non-singular normal mode in general has an irrational number (e.g., Pedlosky, 1987), while that of the singular mode has a rational number as $c^{i}=\lambda(2 i-1) /(2 N)$ for $i=2,3, \cdots$, $N-1$, irrespective of zonal wavenumber $k$. This value corresponds to the basic zonal flow at the interior model level $z_{i}$. Thus, two eigenvalues do not

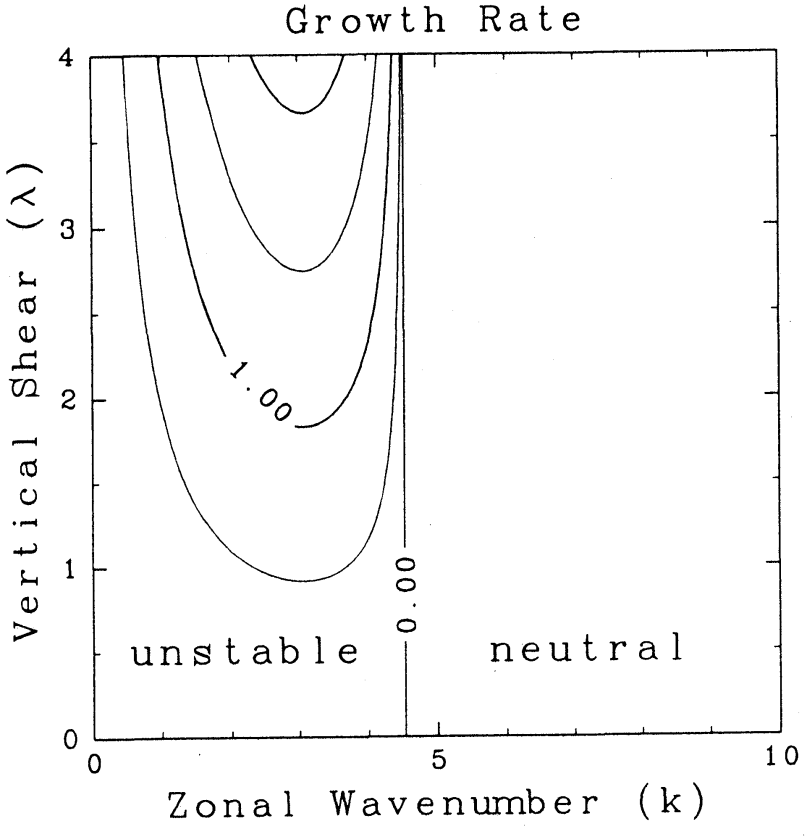

Fig. 2. Stability diagram of the Eady model. The abscissa indicates the zonal wavenumber $k$ and the ordinate the vertical shear $\lambda$ of the basic flow.

coalesce in general, and the solution for the initial value problem can be described by the modal decomposition of (12).

\section{c. Growth rate of the optimal perturbation}

Figure 3 shows the growth rate of the optimal perturbation for short (2.3 days) and rather long (6.9 days) time intervals. At first glance, we notice that the perturbation grows even in the neutral region $(k \geq 4.53)$ (see also Fig. 2). It is also found that the growth rate of the optimal perturbation for a given vertical shear $\lambda$ and zonal wavenumber $k$ is larger than that of the unstable mode. The broken lines indicate the wavenumber which gives the maximum growth for a specified vertical shear. For a short time interval or small vertical shear region, the perturbation with a large zonal wavenumber attains the maximum growth rate. However, with the increase of time interval $\tau$ or vertical shear $\lambda$, the most preferred scale for the optimal excitation gradually approaches to the wavenumber of the most unstable mode $(k=3.05)$.

Figure 4 shows an example of the full spectra of the growth rates $\ln \Gamma_{i} /(2 \tau)$ for a time interval of $\tau=3$. There are 100 eigenvalues $\Gamma_{i}$ for the 50-layer model. The dimension of the subspace of the growing perturbation is a half of the dimension of the system $\left(\ln \Gamma_{i} /(2 \tau)>0\right.$ for $\left.i \leq 50\right)$. The symmetry of the spectra in this figure indicates that the volume of the ellipsoid is conserved in phase space because this model is a conserved system (Yoden 

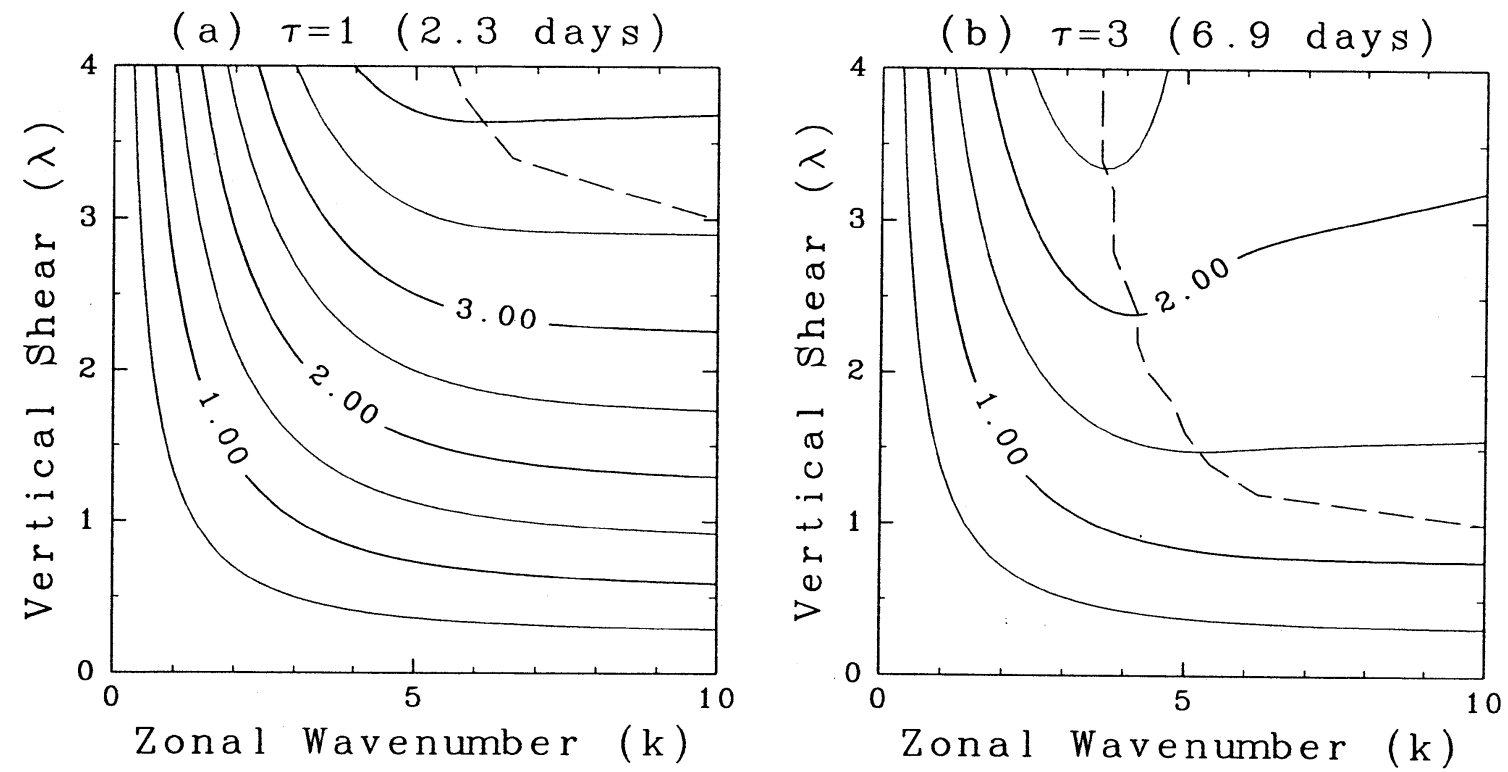

Fig. 3. Growth rate of the optimal perturbation in the 50-layer model for two different time intervals: (a) $\tau=1$ (2.3 days); (b) $\tau=3$ (6.9 days). The abscissa indicates the zonal wavenumber $k$ and the ordinate the vertical shear $\lambda$ of the basic flow. Broken lines denote the wavenumber which gives the maximum growth rate for a specified vertical shear.

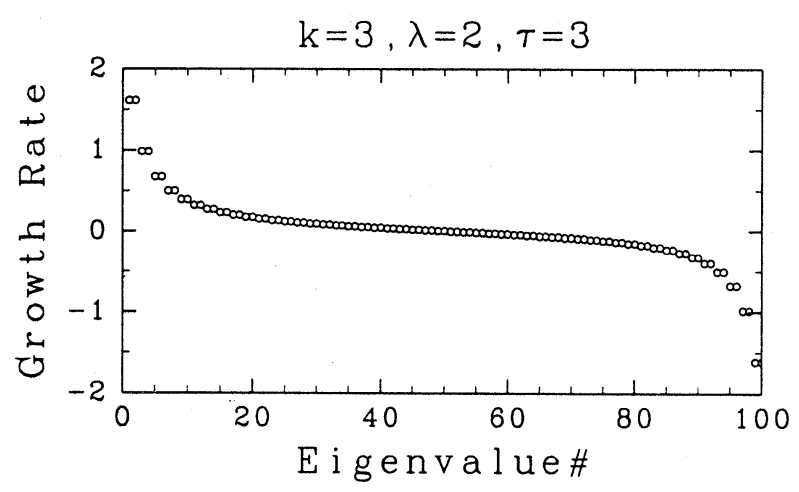

Fig. 4. Spectra of the growth rate for all modes with a time interval of $\tau=3$ $(k=3, \lambda=2)$.

and Nomura, 1993). It is also noted that all spectra are classified into 50 pairs, each of which has the same growth rate. This is because the perturbation streamfunction is represented by the cosine and sine components in (16). A pair of modes with the same growth rate have the same spatial wave structure, but are different in phase of $\pi / 2$ in the zonal direction.

In the following subsections, we describe the detailed characteristic of the optimal perturbation in the unstable region represented by $k=3$ and $\lambda=2$, and in the neutral region represented by $k=6$ and $\lambda=2$.

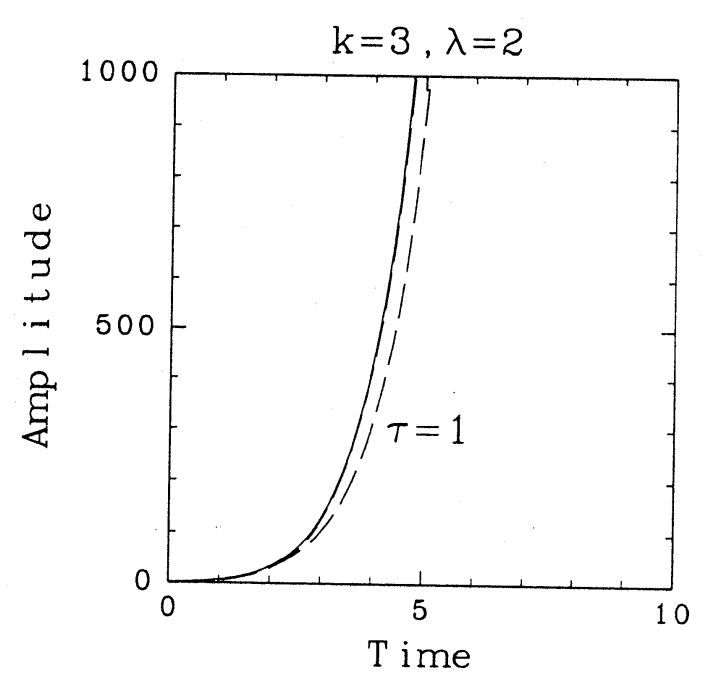

Fig. 5. Growth of the maximum amplitude \| $\boldsymbol{y}(t) \|$ of the optimal perturbation in the linearly unstable region of $k=3$ and $\lambda=2$ (solid line). Broken lines show the respective evolution of the amplitude of the optimal perturbation for three specified time intervals $(\tau=1,3$, and 5$)$.

\section{d. Optimal growth in the unstable region}

Figure 5 shows the temporal evolution of the amplitude $\|\boldsymbol{y}(t)\|$ of the optimal perturbation in the unstable region $(k=3, \lambda=2)$. The broken lines denote the respective evolution of the optimal perturbation for some specified time intervals $(\tau=1$, 3 , and 5), and the solid line indicates the maximum amplitude at each instant. It is found that the 

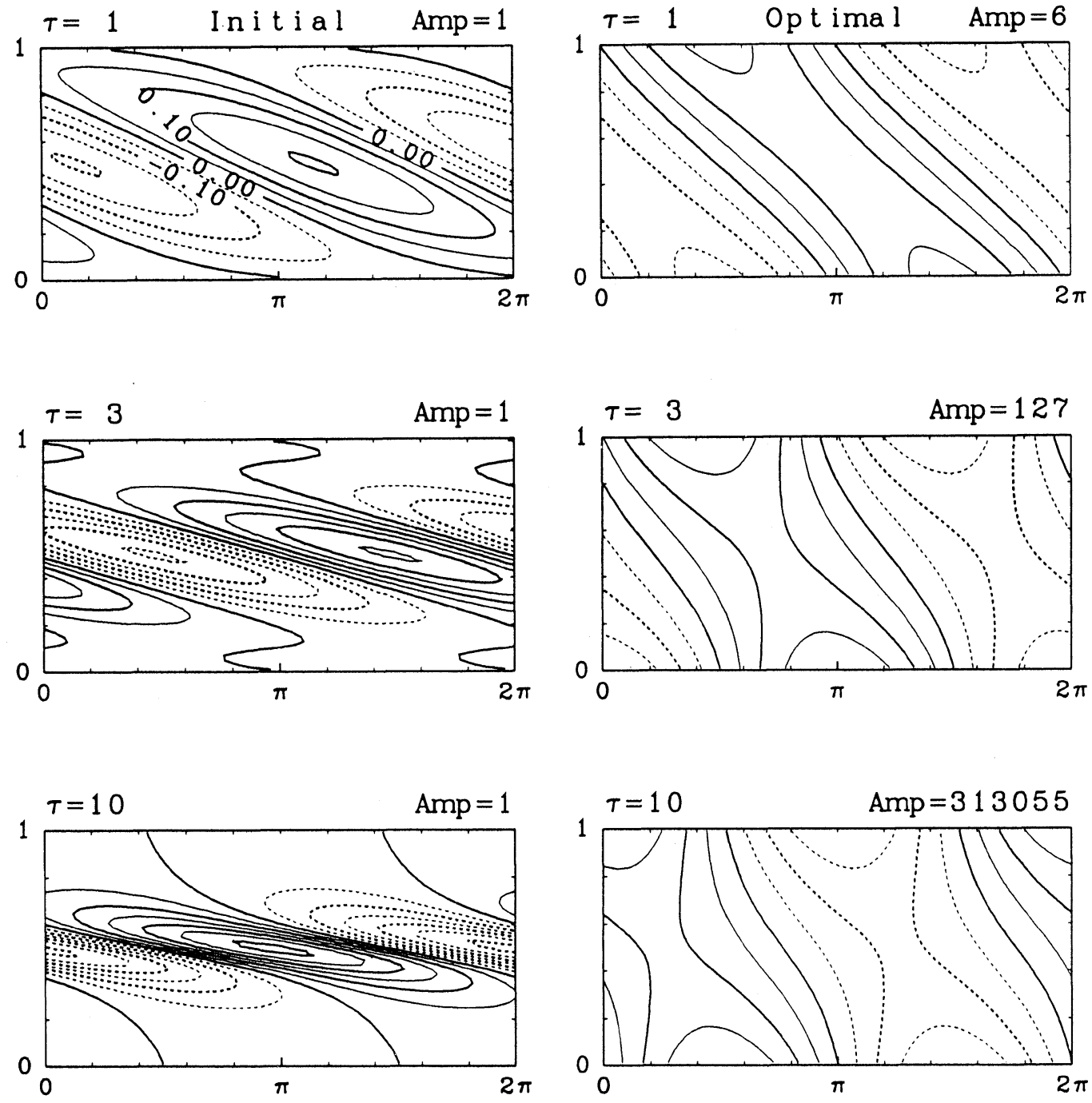

Fig. 6. Streamfunction field of the optimal perturbation at the center of the channel $(y=0)$ in the linearly unstable region $(k=3, \lambda=2)$ for three time intervals $(\tau=1,3$, and 10). The abscissa denotes the $x$-coordinate in one wavelength and the ordinate the $z$-coordinate. The amplitude of the perturbation is normalized to be unity in drawing contours. Left: the initial optimal disturbance. Right: the excited optimal perturbation.

amplitude of the respective optimal perturbation as well as the maximum amplitude grows exponentially in time as the unstable mode.

The vertical structure of the streamfunction field in the center of the channel for the optimal perturbation and the corresponding optimal initial disturbance is shown in Fig. $6(\tau=1,3$, and 10). Note that the norm of $\boldsymbol{y}(t)$ is normalized to be unity in drawing contours, and the phase in the zonal direction is arbitrary in the figure. For a short time interval $(\tau=1)$, the phase line of the optimal perturbation has a larger inclination than that of the unstable normal mode, and is tilting to the vertical shear of the basic flow during the growth. This structure bears a close resemblance to that of the optimal perturbation in the neutral region (see also
Fig. 9).

With the increase of the time interval $\tau$, the excited optimal perturbation gradually gains the familiar unstable normal mode structure, while the corresponding optimal initial disturbance obtains a well defined westward-tilting wave structure confined in small vertical scales. The structure corresponds to the adjoint of the unstable normal mode, which satisfies the adjoint eigenvalue problem of (14) (Farrell, 1989). From (15), the projection coefficient $\alpha^{i}$ of the unstable mode $\phi^{i}$ becomes maximum when the initial disturbance $\hat{\psi}(0)$ coincides with the adjoint $\tilde{\phi}^{i}$ of the mode $\phi^{i}$. Thus, the initial disturbance $\tilde{\phi}^{i}$ most effectively excites the unstable mode $\phi^{i}$.

Temporal evolution of the vertical structure of the 

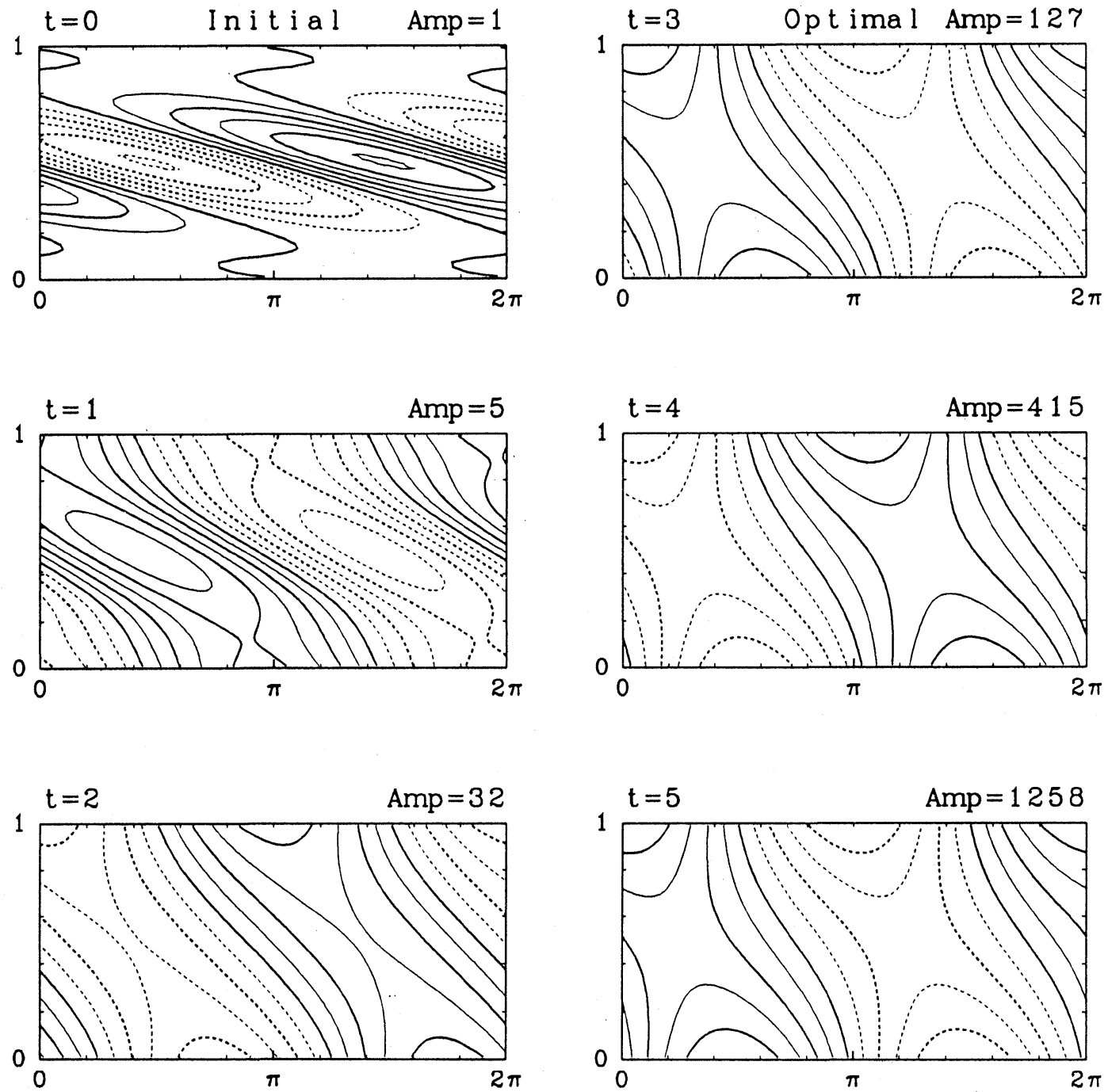

Fig. 7. Evolution of the streamfunction field of the optimal perturbation for the time interval $\tau=3$ at the center of the channel $(y=0)$ in the linearly unstable region $(k=3, \lambda=2)$.

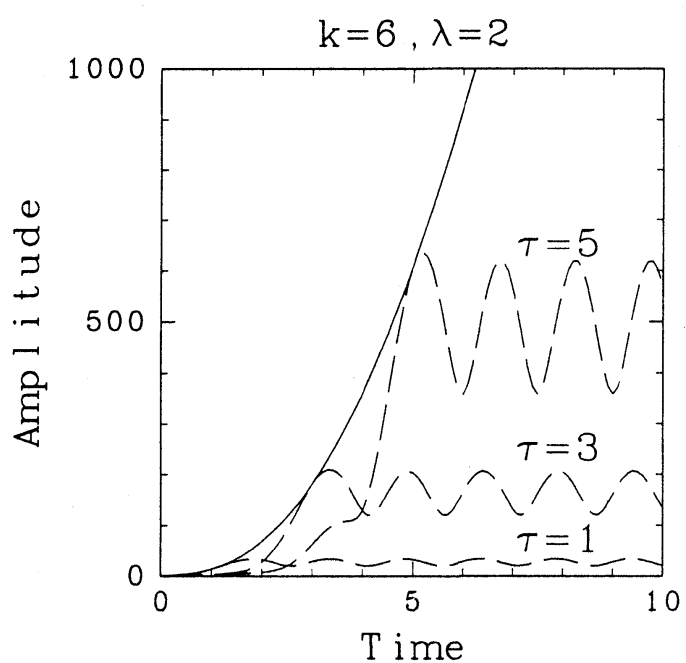

Fig. 8. As in Fig. 5 but for the linearly neutral region $(k=6, \lambda=2)$. optimal perturbation for $\tau=3$ is shown in Fig. 7 . In the initial stage $(t \leq 2)$, the phase line gradually rotates clockwise, and the amplitude near the boundaries becomes large. This feature also resembles the initial development of the optimal perturbation in the neutral region (see also Fig. 11). Thus, for the synoptically relevant time scale, it is suggested that the unstable normal mode does not play a decisive role in the development of the disturbance as might be traditionally accepted (Farrell and Ioannou, 1993). After constructing a typical baroclinic wave structure by $t=3$, the perturbation grows exponentially in time with a fixed baroclinic wave structure.

\section{e. Optimal growth in the neutral region}

The growth of the amplitude $\|\boldsymbol{y}(t)\|$ of the optimal perturbation in the neutral region is shown in Fig. $8(k=6, \lambda=2)$. The maximum amplitude (solid line) shows an algebraic growth which 

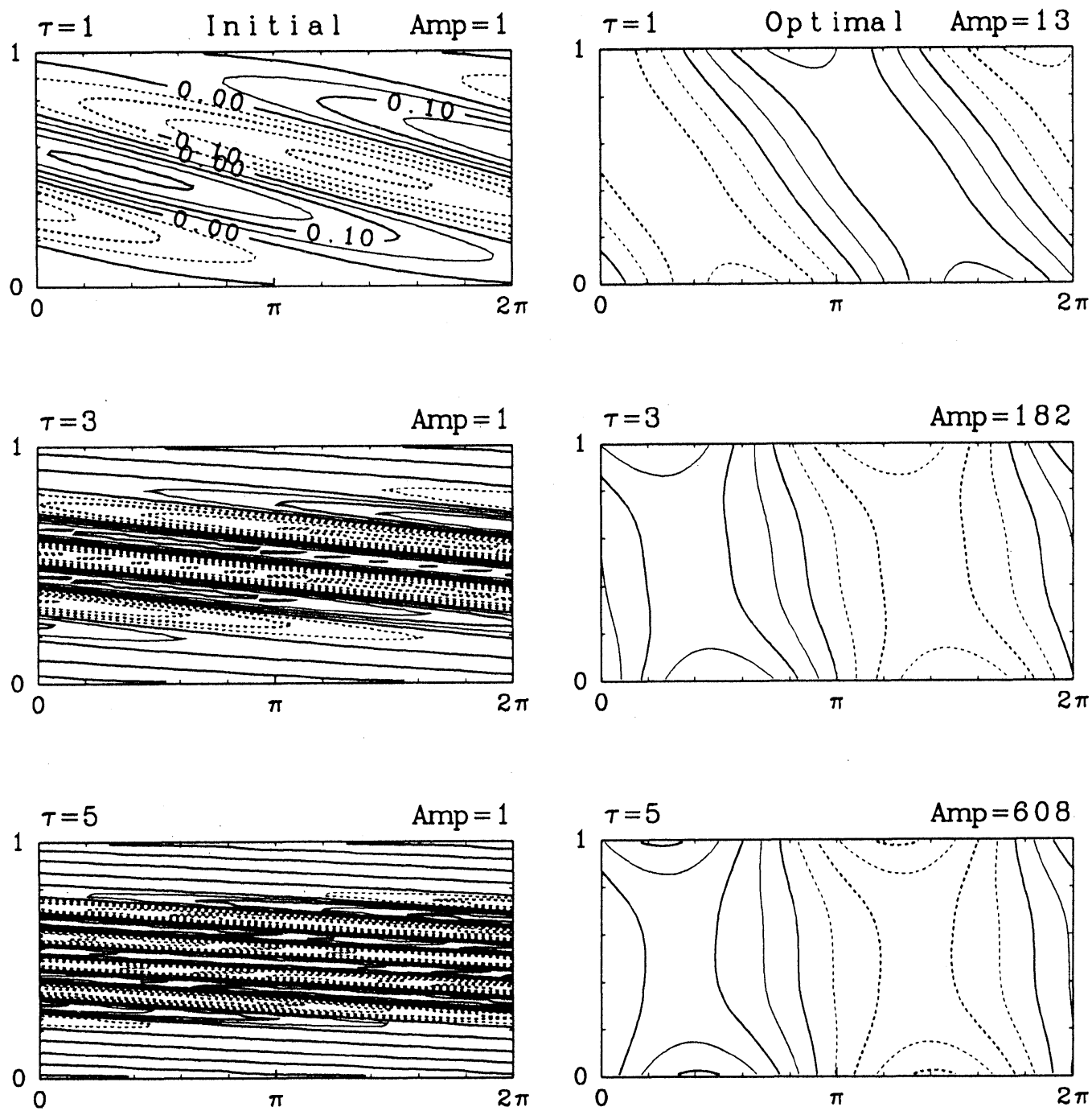

Fig. 9. As in Fig. 6 but for the linearly neutral region $(k=6, \lambda=2)$.

is well expressed by $\|\boldsymbol{y}(\tau)\| \sim C \tau^{2}+1$ where $C$ is a constant (see also Fig. 12b). The amplitude of the respective optimal perturbation for time intervals $\tau=1,3$, and 5 (broken lines) oscillates in time after attaining the maximum growth, which is totally different from that in the unstable region in Fig. 5.

Streamfunction fields of the optimal perturbation and the initial disturbance are shown in Fig. 9. For a small time interval $(\tau=1)$, the wave structure is similar to that in the unstable region (see Fig. 6). Although the vertical scale of the initial disturbance in Fig. 9 is half the size of that in Fig. 6 for $\tau=1$, the westward inclination of the phase line to the vertical is the same since the zonal wavenumber of the former is twice as large as that of the latter. With the increase of the time interval $\tau$, however, the westward inclination of the phase line of the initial disturbance becomes larger. The vertical scale also becomes smaller and does not converge to a well defined structure as in the unstable region.

This structure is well approximated by the plane wave form of $A \sin (k x+m z)$ with $k, m>0$. Figure 10 shows the dependence of the vertical wavenumber $m$ divided by $2 \pi$ of the optimal initial disturbance on the time interval $\tau$. For small $\tau$, the vertical wavenumber linearly increases with $\tau$. Thus, there is an inevitable limit of the time interval $\tau$ for the layer model with any fine vertical resolution to resolve accurately the fine vertical structure of the initial disturbance. For longer time intervals than $\tau=13$, the 50-layer model cannot describe the accurate evolution of the optimal perturbation as depicted in this figure, since the vertical wavenumber $m /(2 \pi)$ of the optimal initial disturbance becomes larger than 25, which is the maximum vertical wavenumber resolved in the 50-layer model. It is also found that the limit of the time interval $\tau$ decreases with the increase of the vertical shear $\lambda$ or the increase of the zonal wavenumber $k$ 


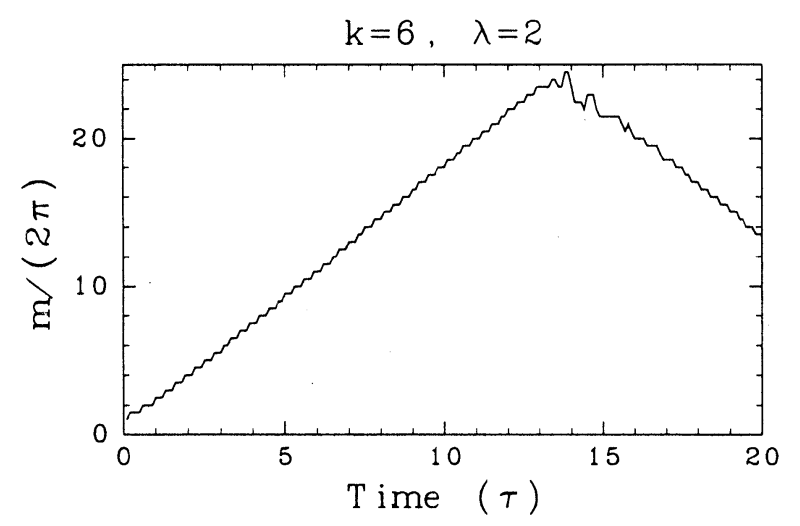

Fig. 10 Dependence of the vertical wavenumber $m /(2 \pi)$ of the optimal initial disturbance on the time interval $\tau(k=6$, $\lambda=2)$.

of the perturbation (not shown). This is intimately related with the convergent property of the growth rate of the optimal perturbation described in Subsection $4 a$.

On the other hand, the excited optimal perturbation in Fig. 9 has the gravest vertical scale with the maximum amplitude near the boundaries, which is irrespective of the time interval $\tau$. A weak westward tilt of the phase line is also noticed. Thus, the optimal perturbation in the neutral region grows by the baroclinic conversion of the available potential energy of the basic flow as the unstable normal mode. It is interesting that the structure of the optimal perturbation resembles that of the unstable mode.

The time evolution of the spatial structure of the optimal perturbation for $\tau=5$ is shown in Fig. 11 . In the initial stage $(t \leq 2)$, the phase line gradually rotates clockwise, and the amplitude near the boundaries becomes large. In this stage, the growth is not energetic (see also Fig. 8). After constructing the baroclinic wave structure by $t=3$, the amplitude shows an explosive growth. When the wave structure becomes almost barotropic at $t=5$, the amplitude attains its maximum value. After this time, the perturbation does not maintain baroclinic wave characteristics as in the unstable region (see Fig. 7). The wave structure is mainly determined by the upper and lower edge wave, each of which has an amplitude maximum near either of the boundaries. Since the upper edge wave propagates faster than the lower edge wave, the vertical tilt of the phase line changes periodically in time and the wave amplitude also vacillates as depicted in Fig. 8. Since the energy source for the growth of the perturbation is the available potential energy of the basic shear flow, the change of the vertical phase tilt, which is intimately related with the baroclinic conversion, produces the amplitude vacillation.
By considering the phase speed, it is revealed that these edge waves are the neutral non-singular normal modes. Because each mode is not orthogonal to the others in our definition of the inner product by (13), these two neutral modes excite a vacillation cycle with a period given by $T=2 \pi /(k \Delta c)$, where $\Delta c$ is the difference in phase speed of the two modes (Farrell, 1982). For the parameter value of Fig. 11, the two neutral normal modes have phase speeds of $c^{1}=1.35$ and $c^{2}=0.65$, resulting in a period $T=2 \pi /(6 \times 0.7)=1.50$ as may be verified by examining Fig. 8 . Therefore, the interference between the two neutral normal modes is responsible for the amplitude vacillation.

\section{Discussion}

In this section, we will discuss some properties of the growing perturbation in the neutral region. First of all, we address the mechanism for the growth of the perturbation by using the Orr mechanism (Orr, 1907; Boyd, 1983; Lindzen, 1990). The key factor for this mechanism is the conservation of the perturbation potential vorticity $q$ of (6) in the interior region of the model, which is given by (5). Thus, if the initial configuration of $q(x, y, z, 0)$ is given by

$$
q(x, y, z, 0)=F(x, z) \cos l_{n} y,
$$

where $F(x, z)$ is an arbitrary function, $q(x, y, z, t)$ at time $t$ is written as

$$
q(x, y, z, t)=F(x-\lambda z t, z) \cos l_{n} y,
$$

which simply states that $q$ is advected by the mean shear flow at each level.

Then, as the obtained optimal initial disturbance in the neutral region, if we assume that the initial configuration of $q$ has the plane wave form:

$$
F(x, z)=A \sin (k x+m z), \quad k, m>0,
$$

with the phase tilt against the shear, the subsequent evolution of $q$ is written as

$$
q(x, y, z, t)=A \sin \{k x+(m-\lambda k t) z\} \cos l_{n} y \cdot(27
$$

Moreover, if we ignore the two horizontal boundaries of the Eady model at $z=0$ and $z=1$, the perturbation streamfunction $\psi(x, y, z, t)$ is obtained by

$$
\begin{aligned}
\psi(x, y, z, t)= & \frac{-A}{\kappa^{2}+(m-\lambda k t)^{2} / S} \times \\
& \sin \{k x+(m-\lambda k t) z\} \cos l_{n} y
\end{aligned}
$$

where $\kappa^{2}=k^{2}+l_{n}^{2}$. From (28), the following properties of the growth by the Orr mechanism are noticed:

(i) Amplitude of the streamfunction attains its maximum value at $t_{\text {orr }}=m /(\lambda k)$. 

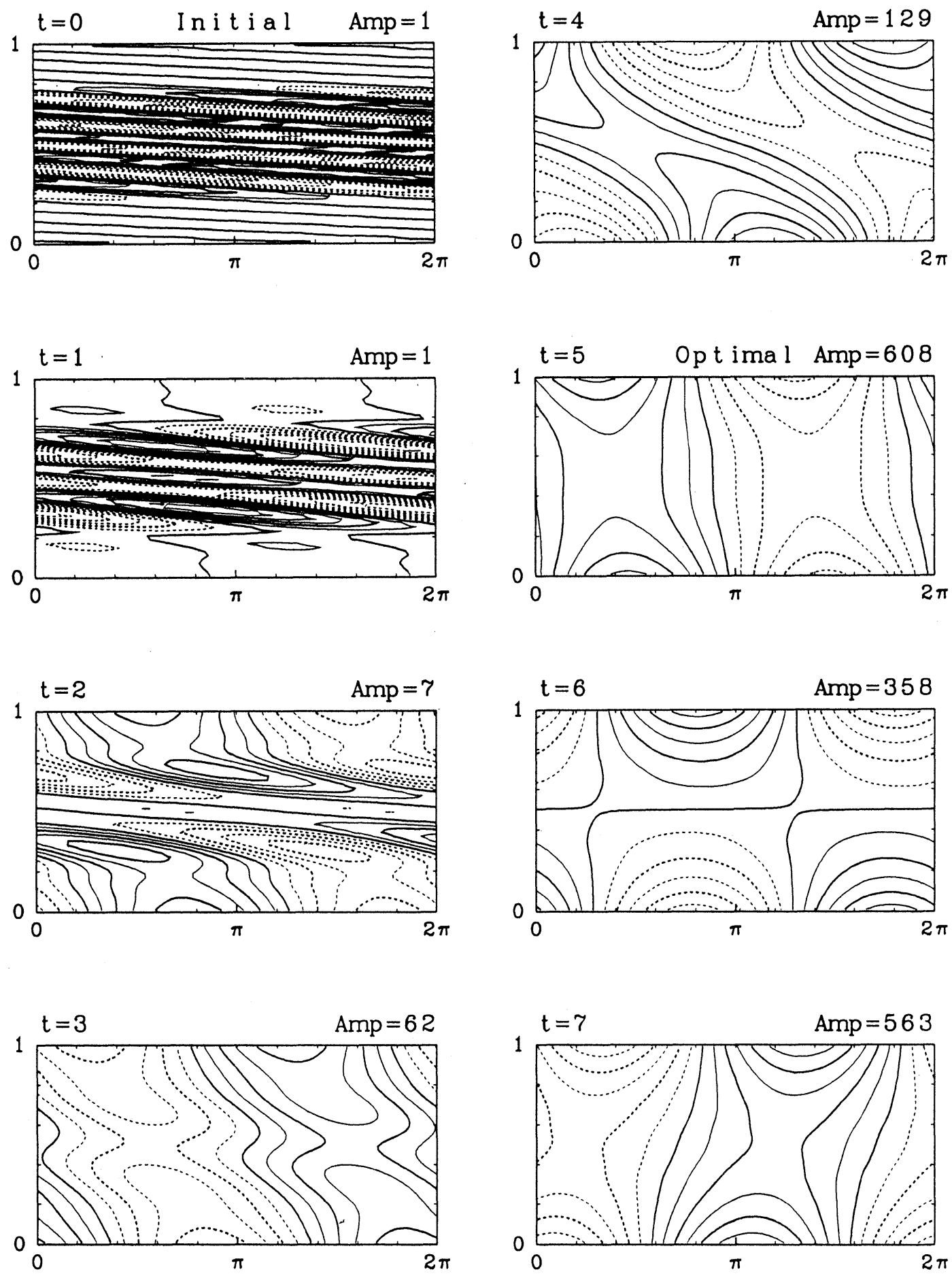

Fig. 11. Evolution of the streamfunction field of the optimal perturbation for the time interval $\tau=5$ at the center of the channel $(y=0)$ in the linearly neutral region $(k=6, \lambda=2)$.

(ii) Maximum amplification of the streamfunction attained at $t_{\text {orr }}$ is given by $A_{\text {orr }}=1+$ $(\tau \lambda k)^{2} /\left(S \kappa^{2}\right)$.

In order to examine whether the Orr mechanism quantitatively describes the growth of the optimal perturbation in the neutral region, the optimization time $\tau$ is compared with $t_{o r r}$ (broken line) in Fig. 12a, while the amplitude $A_{\text {opt }}$ of the opti- mal perturbation at $t=\tau$ (solid line) is compared with $A_{\text {orr }}$ (broken line) in Fig. 12b. The vertical wavenumber of the initial disturbance, from which $t_{\text {orr }}$ is inferred, is obtained from Fig. 10. For the optimization time $\tau \geq 1$ (2.3 days), which is relevant to synoptic scale disturbances, the two lines correspond well with each other in Fig. 12. Thus, the Orr mechanism quantitatively explains well the growth 

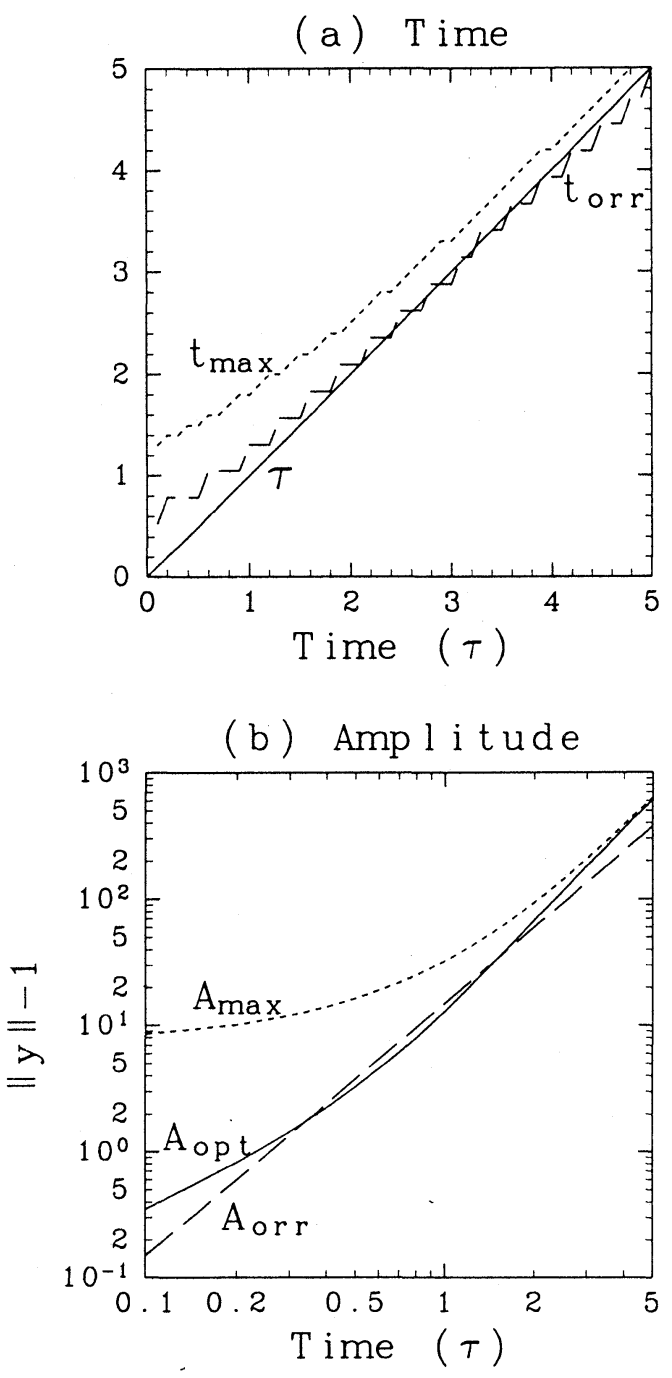

Fig. 12. The maximization time (a) and the maximum amplitude (b) of the perturbation in the neutral region $(k=6$, $\lambda=2)$. Solid lines are the optimization time $\tau$ and the optimal amplitude $A_{\text {opt }}$ at $t=\tau$. Broken lines are the maximization time $t_{\text {orr }}$ and the maximum amplitude $A_{\text {orr }}$ at $t=t_{\text {orr }}$ inferred from the Orr mechanism. Dotted lines are the actual maximization time $t_{\max }$ and the maximum amplitude $A_{\max }$ at $t=t_{\max }$ when the optimal initial disturbance is specified. The ordinate in (b) denotes $\|\boldsymbol{y}\|-1$. See text for details.

of the optimal perturbation in the neutral region.

This result is also very useful to understand various properties of the optimal perturbation in the neutral region. Since the prescribed optimization time $\tau$ corresponds well with $t_{o r r}$, it is easily deduced that the vertical wavenumber $m$ of the optimal initial disturbance is proportional to $\tau$, as depicted in Fig. 10. Moreover, it is also understood that the vertical wavenumber $m$ of the initial disturbance in- creases with the increase of $\lambda$ or $k$ for a specified $\tau$. This in turn explains the convergent property of the growth rate of the optimal perturbation, as denoted in Fig. 1. From the dependence of $A_{\text {orr }}$ on $k$, it is evident that the maximum growth rate of the optimal perturbation attains at high zonal wavenumbers, as shown in Fig. 3.

However, the correspondence with the Orr mechanism may superficially conflict with the results in Farrell (1984): it was shown that the perturbation continues to grow for time intervals twice as long as inferred from the Orr mechanism in the Eady model. In order to clarify this contradiction, the maximization time $t_{\max }$ at which the perturbation attains the maximum amplitude $A_{\max }$ from the specified optimal initial disturbance for each $\tau$ is also plotted in Fig. 12 (dotted lines). Obviously, it is found that $t_{\text {max }}$ is larger than $\tau$ or $t_{\text {orr }}$, and $A_{\max }$ is larger than $A_{\text {opt }}$ or $A_{\text {orr }}$. Farrell (1984) examined the growth of the initial disturbance with the vertical wavenumber $m=\pi$, which roughly corresponds to the optimization time of $\tau=0.1$ in our study from Fig. 10. It also should be noticed that he discussed the development of the perturbation in the unstable region. However, this point makes no matter since the examined time interval was too short for the unstable mode to dominate over the perturbation field. For such a short time interval as $\tau=0.1$, Fig. 12 reveals that $t_{\max }\left(A_{\max }\right)$ is some times larger than $t_{\text {orr }}\left(A_{\text {orr }}\right)$. Thus, it is concluded that the apparent contradiction of our results with Farrell (1984) comes from the difference in time intervals to assess the growth of the perturbation.

The growth by the interference between two nonsingular normal modes, of which existence is due to the presence of the two rigid horizontal boundaries in the Eady model, explains such a large difference between $t_{\max }\left(A_{\max }\right)$ and $t_{\text {orr }}\left(A_{\text {orr }}\right)$ for small $\tau$ in Fig. 12. Since the Orr mechanism will also predict the eventual decay of the perturbation, which is evident from (28), the amplitude vacillation after attaining the optimal growth in Fig. 8 is attributed to the interference.

The predominance of the neutral non-singular normal modes in the excited optimal perturbation, which is responsible for the amplitude vacillation, is explained by considering the modal decomposition. Since the norm of the initial disturbance $\|\hat{\boldsymbol{\psi}}(0)\|$ is assumed to be unity, it is evident from (15) that a particular mode $\phi^{i}$ has a chance to obtain a large amplitude if the projectability $\gamma^{i}$ (Borges and Hartmann, 1992) of this mode defined by

$$
\gamma^{i}=\frac{1}{\left\langle\phi^{i} \cdot \tilde{\phi}^{i}\right\rangle},
$$

has a large value. Thus, in order to effectively excite the perturbation, the initial disturbance $\hat{\boldsymbol{\psi}}(0)$ 


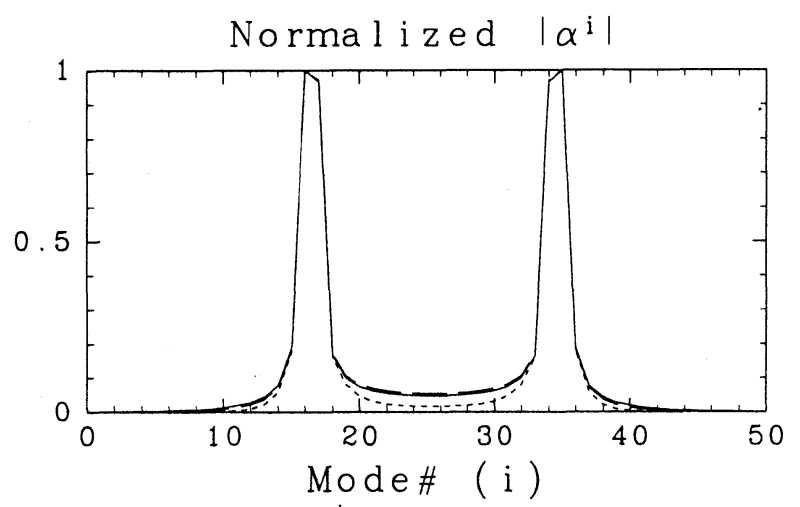

Fig. 13. Absolute value of the projection coefficient $\left|\alpha^{i}\right|$ of the optimal perturbation to each normal mode for three specified time intervals in the linearly neutral region $(k=6, \lambda=2)$ : the solid line is for $\tau=1$; the broken line for $\tau=3$; the dotted line for $\tau=10$. The abscissa denotes the normal mode number in order of the magnitude of the phase speed (mode \#50 has the largest phase speed). The value is normalized for the maximum to be unity.

must have a large projection onto the mode with the largest projectability $\gamma^{i}$.

Figure 13 shows the results of the modal decomposition of the optimal perturbation in the neutral region for $\tau=1,3$, and $10(k=6, \lambda=2)$. These 50 modes are all neutral and in order of the magnitude of the phase speed (mode \#50 has the largest phase speed). Three lines are drawn for each time interval, but they almost merge into each other. Thus, the coefficient $\alpha^{i}$ is almost independent of the time interval $\tau$. Since the Eady model has a symmetry about the middle vertical level at $z=0.5$, the coefficients also have a symmetry, as elucidated in this figure. The modes with the maximum projection coefficient correspond to the non-singular normal modes (mode \#16 and \#35). The others are singular modes.

On the other hand, Fig. 14 indicates the projectability $\gamma^{i}$ for each mode. It is apparent that each non-singular normal mode has a large projectability. Thus, the predominance of the non-singular modes in the optimal perturbation can be attributed to their large projectability. A large projectability corresponds to $\left\langle\phi^{i} \cdot \tilde{\phi}^{i}\right\rangle \approx 0$ from (29), which means that the adjoint mode $\tilde{\phi}^{i}$ is almost perpendicular to the mode $\phi^{i}$ itself. Therefore, another mode $\phi^{j}$ which is almost parallel to the mode $\phi^{i}$ and satisfies the relation $\left\langle\phi^{i} \cdot \tilde{\phi}^{j}\right\rangle \approx 0$ must exist in the eigenmodes, because the adjoint $\tilde{\phi}^{i}$ is perpendicular to the other modes by the bi-orthogonality (13). This

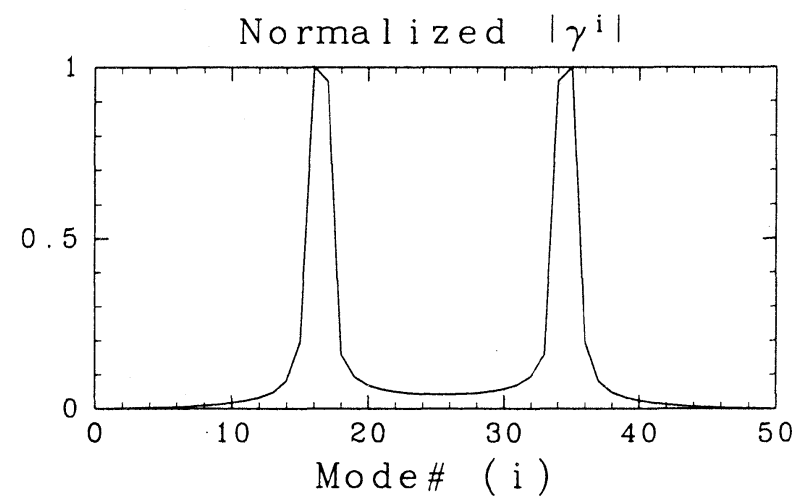

Fig. 14. Same as in Fig. 13 but for the absolute value of the projectability $\left|\gamma^{i}\right|$ of each normal mode $(k=6, \lambda=2)$.

implies that there exist two modes $\phi^{i}$ and $\phi^{j}$ which have a similar vertical structure. In Fig. 14, the existence of the adjacent singular mode (mode \#17 or \#34), which has almost the same phase speed and vertical structure as the corresponding non-singular mode (mode \#16 or \#35), explains the large projectability of the non-singular modes.

\section{Conclusions}

In the framework of the linear theory by using the Eady model, we examined properties of optimal baroclinic perturbations which attain the maximum growth over a prescribed time interval $(\tau)$ in connection with the skill forecast problem in NWPs. The development of the optimal perturbation depends not only on the linear stability of the basic flow, but also on the time interval $\tau$ to assess the growth.

In the linearly unstable region where the perturbation has a small zonal wavenumber, the optimal perturbation grows almost exponentially in time as the unstable normal mode. Its structure gradually coincides with the unstable normal mode as $\tau$ becomes large. The corresponding initial disturbance becomes the adjoint of the unstable mode. For smaller $\tau$ ( $\tau \leq 3$ days), however, the phase line of the optimal perturbation has a larger inclination than the unstable normal mode, and is tilting to the vertical shear of the basic flow during the growth. This is in common with the growth in the neutral region. Thus, it is suggested that the unstable normal mode does not play a significant role in the development of the disturbance for the synoptic time scale.

In the neutral region where the perturbation has a large zonal wavenumber, the maximum amplitude of the optimal perturbation grows as $C \tau^{2}+1$, where $C$ is a constant. However, the amplitude of the respective optimal perturbation for each prescribed time interval vacillates in time after attaining its maximum growth. The optimal initial disturbance has 
a plane wave structure leaning against the shear. The phase line becomes more horizontal and the vertical scale decreases inversely with $\tau$. On the other hand, the excited optimal perturbation has an almost barotropic structure, and is composed mainly of the two neutral non-singular discrete normal modes with different phase speeds; the difference of the phase speed determines the period of the vacillation cycle.

The growth of optimal perturbations in the neutral region is well explained quantitatively by the Orr mechanism for longer time intervals $(\tau \geq 2$ days) to assess the growth. For shorter time intervals, however, the interference between the two non-singular normal modes has a major contribution to the development. The predominance of particular normal modes is also elucidated by the large projectability.

In this simple Eady model in which the basic flow has a constant vertical shear, the initial optimal disturbance has much smaller vertical scale than the excited optimal perturbation. When we consider a more complex basic flow with horizontal as well as vertical shear, the horizontal scale of the optimal initial disturbance will also become small. Thus, it is suggested that a high resolution model which is comparable to the operational full NWP model must be used to resolve the optimal perturbation, and hence to predict accurately the forecast skill of the operational NWP model. So far, however, we have only managed to compute the optimal perturbation in simpler reduced models instead of the full NWP model, even with the present computer resources, since we must solve the eigenvalue problem of a large matrix in the optimization analysis: Kimoto et al., (1992) used a hemispheric barotropic model with T21 truncation (231 variables); Molteni and Palmer (1993) used a T21 barotropic and a three-level quasi-geostrophic global model (483 and 1449 variables, respectively). The structure of the obtained optimal perturbation will be inevitably deformed in these coarse resolution models.

For further studies, it is necessary to reveal the effects of various dissipation mechanisms on the growth of the optimal perturbation. In particular, whether the fine vertical structure of the optimal initial disturbance will fade away by introducing the Newtonian cooling or the vertical diffusion must be elucidated in the next study. We must also clarify the non-linear evolution of the optimal perturbation, which is related with the prediction of the forecast skill in dynamical extended-range forecasts.

\section{Acknowledgments}

We would like to thank Drs. S. Yoden, M. Kimoto, and E. Kalnay for their helpful discussion. We also express our hearty thanks to two anonymous reviewers for their valuable comments on the original manuscript. The computations were performed on SUN SPARCstation ELC of Meteorological College. GFD-DENNOU Library was used for drawing figures.

\section{References}

Bell, M.J. and A.A. White, 1988: Spurious stability and instability in $N$-level quasi-geostrophic models. $J$. Atmos. Sci., 45, 1731-1738.

Borges, M.D. and D.L. Hartmann, 1992: Barotropic instability and optimal perturbations of observed nonzonal flows. J. Atmos. Sci., 49, 335-354.

Boyd, J.P., 1983: The continuous spectrum of linear Couette flow with the beta effect. J. Atmos. Sci., 40, 2304-2308.

Charney, J.G., 1947: The dynamics of long waves in a baroclinic westerly current. J. Meteor., 4, 135-162.

Eady, E.T., 1949: Long waves and cyclone waves. Tellus, 1, 33-52.

Farrell, B.F., 1982: The initial growth of disturbances in a baroclinic flow. J. Atmos. Sci., 39, 1663-1686.

Farrell, B.F., 1984: Modal and non-modal baroclinic waves. J. Atmos. Sci., 41, 668-673.

Farrell, B.F., 1988: Optimal excitation of neutral Rossby waves. J. Atmos. Sci., 45, 163-172.

Farrell, B.F., 1989: Optimal excitation of baroclinic waves. J. Atmos. Sci., 46, 1193-1206.

Farrell, B.F., 1990: Small error dynamics and the predictability of atmospheric flows. J. Atmos. Sci., 47, 2409-2416.

Farrell, B.F. and P.J. Ioannou, 1993: Stochastic forcing of perturbation variance in unbounded shear and deformation flows. J. Atmos. Sci., 50, 200-211.

Held, I.M., 1985: Pseudomomentum and the orthogonality of modes in shear flows. J. Atmos. Sci., 42, 2280-2288.

Kimoto, M., H. Mukougawa and S. Yoden, 1992: Medium-range forecast skill variation and blocking transition: A case study. Mon. Wea. Rev., 120, 1616-1627.

Lacarra, J.-F. and O. Talagrand, 1988: Short-range evolution of small perturbations in a barotropic model. Tellus, 40, 81-95.

Lindzen, R.S., 1990: Dynamics in Atmospheric Physics. Cambridge Univ. Press, 310 pp.

Lorenz, E.N., 1965: A study of the predictability of a 28-variable atmospheric model. Tellus, 17, 321-333.

Molteni, F. and T.N. Palmer, 1993: Predictability and finite-time instability of the northern winter circulation. Quart. J. Roy. Meteor. Soc., 119, 269-298.

Mukougawa, H., M. Kimoto and S. Yoden, 1991: A relationship between local error growth and quasistationary states: Case study in the Lorenz system. J. Atoms. Sci., 48, 1231-1237.

Mureau, R., F. Molteni and T.N. Palmer, 1993: Ensemble prediction using dynamically conditioned perturbations. Quart. J. Roy. Meteor. Soc., 119, 299-323.

Orr, W.McF., 1907: Stability or instability of the steady-motions of a perfect liquid. Proc. Roy. Irish Acad., 27, 9-69. 
Palmer, T.N., 1993: Extended-range atmospheric prediction and the Lorenz model. Bull. Amer. Meteor. Soc., 74, 49-65.

Pedlosky, J., 1964: An initial value problem in the theory of baroclinic instability. Tellus, 16, 12-17.

Pedlosky, J., 1987: Geophysical Fluid Dynamics. Springer-Verlag, $710 \mathrm{pp}$.
Toth, Z. and E. Kalnay, 1993: Ensemble forecasting at NMC: The generation of perturbations. Bull. Amer. Meteor. Soc., 74, 2317-2330.

Yoden, S. and M. Nomura, 1993: Finite-time Lyapunov stability analysis and its application to atmospheric predictability. J. Atmos. Sci., 50, 1531-1543.

\title{
Eady モデルにおける傾圧波の最適励起
}

\author{
向川 均 ${ }^{1}$ 池田 徹 $^{2}$ \\ (気象大学校)
}

数値予報モデルにおける予報誤差予測の問題と関連して、最適励起擾乱の特性を線形論の範囲で Eady モデルを用いて詳しく調べた。最適励起擾乱を、その振幅がある与えられた時間 $(\tau)$ で最大に発達するも のとして定義する。擾乱発達の特性は、基本場の力学的安定性だけではなく、発達を評価する時間 $\tau$ に依 存する。

線形的に不安定な領域では、最適励起擾乱は、不安定モードと同様にほぼ指数関数的に成長する。時間 $\tau$ が増加するに従い、その構造は不安定モードのそれに漸近する。また、対応する初期擾乱は不安定モード の随伴モードとなる。一方、時間 $\tau$ が小さいときには $(\tau \leq 3$ 日)、最適励起擾乱の等位相線の傾きは不安 定モードに比べより水平に近く、不安定モードは擾乱の初期の発達には関与しない。

線形的に中立な領域においては、最適励起摠乱は、 $C \tau^{2}+1$ ( $C$ は定数) のように代数的に成長する。対 応する初期擾乱は、鉛直シアーに対し傾いた平面波の構造を持っている。その等位相線の傾きは時間 $\tau$ の増 加に比例して水平に近づき、擾乱の鉛直スケールは逆比例して減少する。他方、最適励起擾乱はほぼ順圧 的な構造となり、その構造は位相速度の異なる、特異点を持たない 2 つの中立モードにより主として決定 される。発達評価時間 $\tau$ が比較的大きい場合 $(\tau \geq 2$ 日)、最適励起擾乱の発達は、Orr のメカニズムで定

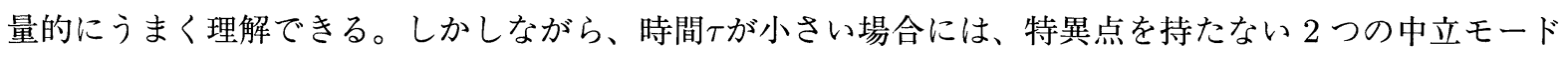
間での干渉が、擾乱の成長に大きく寄与する。また、成長した最適励起擾乱の構造は、各モードの射影性 (projectability) より説明される。

これらの結果は、数值予報モデルの予報誤差予測を行うには、数值予報モデルと同程度の空間解像度を 持つモデルが必要であることを示唆している。

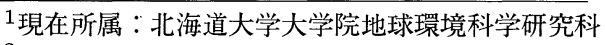

2 現在所属 : 福井地方気象台
} 